Georgian Mathematical Journal

Volume 13 (2006), Number 3, 495-500

\title{
INVERSION OF AHLFORS AND GRUNSKY INEQUALITIES
}

\author{
SAMUEL KRUSHKAL
}

\begin{abstract}
We solve the old Kühnau's problem on the exact lower bound in the inverse inequality estimating the dilatation of a univalent function by its Grunsky norm and in the related Ahlfors inequality for Fredholm eigenvalues.
\end{abstract}

2000 Mathematics Subject Classification: Primary: 30C35, 30C62, 30F45; Secondary: 30F60, 31A10, 53A35.

Key words and phrases: Grunsky coefficient inequalities, Fredholm eigenvalues, quasiconformal reflection, subharmonic function, Strebel point, conformal metric, generalized Gaussian curvature.

1. The Ahlfors inequality for oriented quasiconformal Jordan curves (quasicircles) on the Riemann sphere $L \subset \widehat{\mathbb{C}}=\mathbb{C} \cup\{\infty\}$

$$
\frac{1}{\rho_{L}} \leq q_{L}
$$

is fundamental in the theory of Fredholm eigenvalues. Here $q_{L}$ is the reflection coefficient of $L$ and $\rho_{L}$ is its (first nontrivial) Fredholm eigenvalue (see, e.g., [1], $[2],[6],[9])$.

It suffices to take the images $L=f^{\mu}\left(S^{1}\right)$ of the unit circle $S^{1}$ under quasiconformal self-maps of $\widehat{\mathbb{C}}$ with Beltrami coefficients $\mu(z)=\partial_{\bar{z}} f / \partial_{z} f$ supported in the unit disk $\Delta=\{z:|z|<1\}$ and hydrodynamic normalization $f(z)=z+$ const $+O\left(|z|^{-1}\right)$ at $z=\infty$. Then $q_{L}$ equals a minimal dilatation $k\left(f^{\mu}\right)=\|\mu\|_{\infty}$ among such maps, and inequality (1.1) is reduced to the Grunsky inequality

$$
\varkappa(f):=\sup _{\mathbf{x}}\left|\sum_{m, n=1}^{\infty} \sqrt{m n} \alpha_{m n} x_{m} x_{n}\right| \leq k(f),
$$

where $\alpha_{m n}$ are the Grunsky coefficients of $f$ defined by

$$
\log \frac{f(z)-f(\zeta)}{z-\zeta}=-\sum_{m, n=1}^{\infty} \alpha_{m n} z^{-m} \zeta^{-n}, \quad(z, \zeta) \in\left(\Delta^{*}\right)^{2},
$$

and $\mathbf{x}=\left(x_{n}\right)$ runs over the unit sphere $S\left(l^{2}\right)$ of the Hilbert space $l^{2}$ with $\|\mathbf{x}\|^{2}=\sum_{1}^{\infty}\left|x_{n}\right|^{2}$, choosing the principal branch of logarithmic function (cf. [4], $[9])$. The quantity $\varkappa(f)$ is called the Grunsky norm of $f$. By Kühnau-Schiffer theorem $\varkappa(f)$ is reciprocal of $\rho_{L}$ (see [10], [13]). 
We denote the collection of univalent nonvanishing functions $f(z)=z+b_{0}+$ $b_{1} z^{-1}+\ldots$ by $\Sigma$ and its subset of functions with quasiconformal extensions across $S^{1}$ by $\Sigma^{0}$.

The point is that for most of $f \in \Sigma$, we have in (1.2) the strict inequality $\varkappa(f)<k(f)$ (see, e.g., [8]). On the other hand, the functions with $\varkappa(f)=k(f)$ are crucial in many applications of the Grunsky inequality technique. Moreover, by theorem of Pommerenke and Zhuravlev, any $f \in \Sigma$ with $\varkappa(f) \leq k<1$ belongs to $\Sigma^{0}$ and has a $k_{1}$-quasiconformal extension with $k_{1}=k_{1}(k) \geq k$ (see [11], [7], pp. 82-84). An explicit not sharp bound $k_{1}(k)$ is given in [10].

The important problem on the sharp estimation of the dilatation $k(f)$ by the Grunsky norm of $f$, or equivalently, by the Fredholm eigenvalue of $f\left(S^{1}\right)$ was first stated by Kühnau in 1981 and still remains open. Our main result is the following theorem which solves this problem and has many other applications.

Theorem 1. For $f \in \Sigma^{0}$ we have the estimate

$$
k(f) \leq \frac{3}{2 \sqrt{2}} \varkappa(f)=1.07 \ldots \varkappa(f)
$$

(similarly for Fredholm eigenvalues $\left.\rho_{f\left(S^{1}\right)}\right)$, which is asymptotically sharp as $\varkappa \rightarrow 0$. The equality holds for the map

$$
f_{3, t}(z)= \begin{cases}z\left(1+t / z^{3}\right)^{2 / 3} & \text { if }|z|>1 \\ z\left[1+t(|z| / z)^{3}\right]^{2 / 3} & \text { if }|z| \leq 1\end{cases}
$$

with $t=$ const $\in(0,1)$.

Note that the Beltrami coefficient of this map in the disk $\Delta$ is $\mu_{3}(z)=t|z| / z$.

2. The proof of this theorem consists of several independent stages which will be outlined below.

$\mathbf{1}^{\mathbf{0}}$. It suffices to establish the assertion of Theorem 1 for $f \in \Sigma^{0}$ having Teichmüller extremal quasiconformal extensions onto $\Delta$, i.e., with the Beltrami coefficient $\mu_{f}(z)=k|\varphi(z)| / \varphi(z)$, where $k=$ const $\in(0,1)$ and $\varphi$ is integrable holomorphic function in $\Delta$. This means that $f$ is represented in the universal Teichmüller space $\mathbf{T}$ by a Strebel point $[f]$. Such points are dense in $\mathbf{T}$ (see [3], [14]).

Recall that $\mathbf{T}$ is the space of quasisymmetric homeomorphisms of the unit circle $S^{1}$ factorized by Möbius maps. It inherits a complex Banach structure factorizing the ball of conformal structures

$$
\operatorname{Belt}(\Delta)_{1}=\left\{\mu \in L_{\infty}(\mathbb{C}): \mu \mid \Delta^{*}=0,\|\mu\|<1\right\}
$$

on $\Delta$ so that $\mu, \nu \in \operatorname{Belt}(\Delta)_{1}$ are equivalent if the corresponding maps $w^{\mu}, w^{\nu} \in$ $\Sigma^{0}$ coincide on $S^{1}$. The equivalence classes are in the one-to-one correspondence with the Schwarzian derivatives $\left.S_{f}:=\left(w^{\prime \prime} / w^{\prime}\right)^{\prime}-w^{\prime \prime} / w^{\prime}\right)^{2} / 2$ of $f \in \Sigma^{0}$ in the complementary disk $\Delta^{*}=\{z \in \widehat{\mathbb{C}}:|z|>1\}$. 
For elements $\mu \in \operatorname{Belt}(\Delta)_{1}$ we define

$$
\langle\mu, \varphi\rangle_{\Delta}=\iint_{\Delta} \mu_{0}^{*}(z ; f) \psi(z) d x d y, \quad \varphi \in L_{1}(\Delta),
$$

and put $\mu^{*}(z)=\mu(z) /\|\mu\|_{\infty}$ so that $\left\|\mu^{*}\right\|_{\infty}=1$, and $\|\varphi\|_{1}:=\|\varphi\|_{L_{1}(\Delta)}$.

$\mathbf{2}^{\mathbf{0}}$. We first prove

Theorem 2. For every function $f \in \Sigma^{0}$ with a unique extremal extension $f^{\mu_{0}}$ to $\Delta$, we have the sharp bound

$$
k\left(f^{\mu_{0}}\right) \leq \frac{1}{\alpha\left(f^{\mu_{0}}\right)} \min _{|t|=1} \varkappa\left(f^{t \mu_{0}}\right)
$$

with

$$
\alpha\left(f^{\mu_{0}}\right)=\sup _{\psi \in A_{1}^{2},\|\varphi\|_{A_{1}=1}}\left|\left\langle\mu_{0}^{*}, \varphi\right\rangle_{\Delta}\right|
$$

where

$$
A_{1}^{2}=\left\{\varphi \in L_{1}(\Delta): \varphi=\psi^{2}, \psi \text { is holomorphic }\right\} .
$$

Proof of Theorem 2 is geometric and relies on certain deep properties of conformal (semi)metrics $d s=\lambda(z)|d z|$ on the disk $\Delta$ with $\lambda(z) \geq 0$ of negative integral curvature bounded from above. The curvature is understood in the supporting sense of Ahlfors or, more generally, in the potential sense of Royden (see, e.g., [12]). For such metrics we have

Lemma 3 ([12]). If a circularly symmetric conformal metric $\lambda(|z|)|d z|$ in $\Delta$ has curvature at most -4 in the potential sense, then $\lambda(r) \geq a\left(1-a^{2} r^{2}\right)$, where $a=\lambda(0)$.

On the extremal disk

$$
\Delta\left(\mu_{0}^{*}\right)=\left\{\phi_{\mathbf{T}}\left(t \mu_{0}^{*}\right): t \in \Delta\right\} \subset \mathbf{T},
$$

where $\phi_{\mathbf{T}}$ denotes the projection $\operatorname{Belt}(\Delta)_{1} \rightarrow \mathbf{T}$, the infinitesimal KobayashiTeichmüller metric $\lambda_{\mathcal{K}}$ of $\mathbf{T}$ is isometrically equivalent to hyperbolic metric $d s=|d z| /\left(1-|z|^{2}\right)$ on $\Delta$ of curvature -4 .

Further, the Grunsky coefficients of $f \in \Sigma^{0}$ allows us to construct the holomorphic maps

$$
\widetilde{h}_{\mathbf{x}}(t):=h_{\mathbf{x}}\left(\varphi_{t}\right)=\sum_{m, n=1}^{\infty} \sqrt{m n} \alpha_{m n}\left(\varphi_{t}\right) x_{m} x_{n}: \Delta \rightarrow \Delta,
$$

where $\varphi_{t}=S_{f^{t} \mu_{0}^{*}}$ and again $\mathbf{x}=\left(x_{n}\right) \in S\left(l^{2}\right)$. Then $\sup \left\{\left|\widetilde{h}_{\mathbf{x}}(t)\right|: \mathbf{x} \in S\left(l^{2}\right)\right\}=$ $\varkappa\left(f^{\mu_{0}^{*}}\right)$. Pull-backing the hyperbolic metric to $\Delta\left(\mu_{0}^{*}\right)$ by applying these maps, we get the conformal metrics

$$
\lambda_{\widetilde{h}_{\mathbf{x}}}(t):=\widetilde{h}_{\mathbf{x}}^{*}\left(\lambda_{\mathrm{hyp}}\right)=\left|\widetilde{h}_{\mathbf{x}}^{\prime}(t)\right| /\left(1-\left|\widetilde{h}_{\mathbf{x}}(t)\right|^{2}\right)
$$


of Gaussian curvature -4 at noncritical points. Take their upper envelope $\widetilde{\lambda}_{\varkappa}(t)=\sup \left\{\lambda_{\widetilde{h}_{\mathbf{x}}}(t): \mathbf{x} \in S\left(l^{2}\right)\right\}$ and pass to the upper semicontinuous regularization

$$
\lambda_{\varkappa}(t)=\limsup _{t^{\prime} \rightarrow t} \widetilde{\lambda}_{\varkappa}\left(t^{\prime}\right)
$$

This yields a logarithmically subharmonic metric on $\Delta$ whose curvature in the supporting and in the potential sense both are less than or equal -4 . Its circular mean

$$
\mathcal{M}\left[\lambda_{\varkappa}\right](|t|)=(2 \pi)^{-1} \int_{0}^{2 \pi} \lambda_{\varkappa}\left(r e^{i \theta}\right) d \theta
$$

is a circularly symmetric metric with curvature also at most -4 in the potential sense.

To calculate the value of $\mathcal{M}\left[\lambda_{\varkappa}\right](0)$, one can apply the standard variational method to the maps $f^{\mu} \in \Sigma^{0}$ and to their Grunsky coefficients, which yields

$$
\mathcal{M}\left[\lambda_{\varkappa}\right](0)=\lambda_{\varkappa}(0)=\alpha\left(f^{\mu_{0}}\right) .
$$

Further, applying Lemma 3, we get

$$
\mathcal{M}\left[\lambda_{\varkappa}\right](r) \geq \frac{\alpha\left(f_{0}\right)}{1-\alpha\left(f_{0}\right)^{2} r^{2}}
$$

and, integrating both sides of this inequality over a radial segment $[0, \varrho]$ with $\varrho=\left\|\mu_{0}\right\|_{\infty}$

$$
\begin{aligned}
\int_{0}^{\varrho} \mathcal{M}\left[\lambda_{\varkappa}\right](r) d r \geq \tanh ^{-1}\left[\alpha\left(f^{\mu_{0}}\right) \varrho\right] & =\tanh ^{-1}\left[\alpha\left(f^{\mu_{0}}\right) k\left(f^{\varrho \mu_{0}^{*}}\right)\right] \\
& =\tanh ^{-1}\left[\alpha\left(f^{\mu_{0}}\right) k\left(f^{\mu_{0}}\right)\right] .
\end{aligned}
$$

On the other hand, since the disk $\Delta\left(\mu_{0}^{*}\right)$ is geodesic, we have

$$
\int_{0}^{t} \lambda_{\varkappa\left(f^{\left.\mu_{0}\right)}\right.}(t)|d t|=\tanh ^{-1}\left[\varkappa\left(f^{\mu_{0}}\right)\right] .
$$

Using these relations, one obtains the desired estimates (5), (6).

$\mathbf{3}^{\mathbf{0}}$. To get (3), we have to estimate (6) from below. To this end, we apply the following important result.

Lemma 4 ([5], [6]). The equality $\varkappa(f)=k(f)$ for a function $f \in \Sigma$ holds if and only if $f$ is the restriction to $\Delta^{*}$ of a quasiconformal self-map $w^{\mu_{0}}$ of $\widehat{\mathbb{C}}$ with Beltrami coefficient $\mu_{0}$ satisfying the condition

$$
\sup \left|\left\langle\mu_{0}, \varphi\right\rangle_{\Delta}\right|=\left\|\mu_{0}\right\|_{\infty}
$$

where the supremum is taken over holomorphic functions $\varphi \in A_{1}^{2}(\Delta)$ with $\|\varphi\|_{1}=1$. 
If, in addition, the class $[f]$ contains a frame map (is a Strebel point), then $\mu_{0}$ is of the form

$$
\mu_{0}(z)=\left\|\mu_{0}\right\|_{\infty}\left|\psi_{0}(z)\right| / \psi_{0}(z) \text { with } \psi_{0} \in A_{1}^{2} \text { in } \Delta .
$$

For analytic curves $f\left(S^{1}\right)$, the equality (8) is given also in [10].

In view of this lemma and Theorem 2 , we can restrict ourselves in the proof of (3) to finding a minimal value of the functionals $l_{\mu}(\psi)=\left|\left\langle\mu^{*}, \varphi\right\rangle_{\Delta}\right|$ on the set $\left\{\varphi \in A_{1}^{2},\|\varphi\|_{1}=1\right\}$ for $\mu^{*}=|\psi| / \psi$ defined by integrable holomorphic functions in $\Delta$ of the form

$$
\psi(z)=z^{m}\left(c_{0}+c_{1} z+\cdots\right), \quad m=1,3,5, \ldots .
$$

A long complicate evaluation yields that this minimum equals $\frac{2 \sqrt{2}}{3}$ and is attained on the map (4).

\section{REFERENCES}

1. L. V. Ahlfors, Remarks on the Neumann-Poincaré integral equation. Pacific J. Math. 2(1952), 271-280.

2. D. GaIER, Konstruktive Methoden der konformen Abbildung. Springer Tracts in Natural Philosophy, Vol. 3. Springer-Verlag, Berlin, 1964.

3. F. P. Gardiner and N. LAKIC, Quasiconformal Teichmüller theory. Mathematical Surveys and Monographs, 76. American Mathematical Society, Providence, RI, 2000.

4. H. GRUnsky, Koeffizientenbedingungen für schlicht abbildende meromorphe Funktionen. Math. Z. 45(1939), No. 1, 29-61.

5. S. L. Krushkal, Grunsky coefficient inequalities, Carathéodory metric and extremal quasiconformal mappings. Comment. Math. Helv. 64(1989), No. 4, 650-660.

6. S. L. Krushkal, Quasiconformal extensions and reflections. Handbook of complex analysis: geometric function theory. Vol. 2, 507-553, Elsevier, Amsterdam, 2005.

7. S. L. KRUshkal and R. KühnaU, Quasikonforme Abbildungen - neue Methoden und Anwendungen. Teubner-Texte zur Mathematik [Teubner Texts in Mathematics], 54. BSB B. G. Teubner Verlagsgesellschaft, Leipzig, 1983.

8. S. L. KRushKal and R. KühnAU, Grunsky inequalities and quasiconformal extension. Israel J. Math. 152(2006), 49-59.

9. R. KüHnau, Möglichst konforme Spiegelung an einer Jordankurve. Jahresber. Deutsch. Math.-Verein. 90(1988), No. 2, 90-109.

10. R. KühnaU, Über die Grunskyschen Koeffizientenbedingungen. Ann. Univ. Mariae Curie-Sktodowska Sect. A 54(2000), 53-60.

11. Chr. Pommerenke, Univalent functions. Studia Mathematica/Mathematische Lehrbücher, Band XXV. Vandenhoeck \& Ruprecht, Göttingen, 1975.

12. H. L. Royden, The Ahlfors-Schwarz lemma: the case of equality. J. Analyse Math. 46(1986), 261-270.

13. M. Schiffer, Fredholm eigenvalues and Grunsky matrices. Ann. Polon. Math. 39(1981), 149-164.

14. K. Strebel, On the existence of extremal Teichmueller mappings. J. Analyse Math. 30(1976), 464-480.

(Received 25.07.2006) 
Author's addresses:

Department of Mathematics

Bar-Ilan University

52900 Ramat-Gan

Israel

Department of Mathematical sciences

University of Memphis

Memphis, TN 38152

USA

E-mail: skrushkl@memphis.edu 